\title{
Pseudoepitheliomatous Hyperplasia Treated by Photodynamic Therapy with Variable Irradiation Dose and Concentration of Photosensitizer
}

\author{
Qiang Li, M.D., Ph.D., Bin Jiao, M.D., ${ }^{2}$ and Heather Ann Long, Ph.D. ${ }^{3}$
}

\begin{abstract}
Objective: The aim of this study was to test the effectiveness of photodynamic therapy (PDT) in treating pseudoepitheliomatous hyperplasia (PEH) after skin wounding. Background Data: PEH is a difficult-to-treat extreme-degree acanthosis characterized by proliferation of the epithelium. Topical PDT offers an effective and non-invasive treatment for intraepithelial neoplasia and inflammatory dermatosis. These disorders and PEH show the same histological features: epidermal hyperplasia. To our knowledge, there have been no clinical trials published about therapeutic responses of PDT for PEH. Materials and Methods: After application of 10-30\% methyl aminolaevulinate (MAL) emulsion, each lesion was irradiated with 633-nm red light at a total dose of 113-339 J/ $\mathrm{cm}^{2}$. Therapeutic response was assessed by clinical examination at 3 months. Results: Only 4 of 16 lesions clinically showed a minimal response. No response was observed in 12 of the 16 lesions, either with different cumulative doses or different concentrations of MAL. Conclusion: PEH after skin wounding responds poorly to the topical MAL-PDT. Besides removal of underlying diseases, surgical excision is still the recommended first option.
\end{abstract}

\section{Introduction}

$\mathbf{P}$ SEUdoepitheliomatous HYPERPLASIa (PEH) is a difficult-to-treat extreme-degree acanthosis characterized by preparative, hyperplastic process of the epidermis. The histological formation is the outcome of abnormal and reactive epithelial proliferation that occurs in response to underlying infectious, inflammatory, neoplastic conditions and chronic cutaneous wounds. ${ }^{1}$ Conventional therapy for PEH is extensive surgical excision, which can lead to poor cosmetic outcomes, especially when local flaps or skin grafts are required for reconstruction. Topical photodynamic therapy (PDT) offers an effective and non-invasive treatment with good cosmetic outcome for intraepithelial neoplasia and inflammatory dermatosis, such as psoriasis and viral warts. ${ }^{2-4}$ PDT with 10-20\% Methyl aminolaevulinate (MAL) has been reported in successfully treating periungual warts and superficial squamous cell carcinoma (SCC), and has been approved in treating actinic keratosis (AK) and Bowen's disease (BD) that are intraepithelial SCC. ${ }^{5-7}$ These disorders and PEH share similar histological features, namely epidermal hyperplasia. Both atypical cells and proliferating epithelial cells can selectively concentrate the photosensitizing agents, which may trigger photodynamic reactions or secondary effects.
MAL, a novel photosensitizer precursor, showed deeper tumour penetration and fewer adverse effects than 5aminolaevulinic acid (ALA) due to the enhanced lipophilicity. To our knowledge, there has been no clinical trial to date of topical MAL-PDT therapy in the treatment of PEH. Therefore, to test the effectiveness of PDT, we performed this clinical trial involving a case series.

\section{Case Report}

After obtaining ethics committee approval and patients' informed consent, three patients with 16 lesions were enrolled. The diagnosis of PEH was confirmed by at least a 5$\mathrm{mm}$ incision biopsy taken from the thickest part of the lesion. Topical 5-30\% (w/w) MAL (Biosynth AG, Staad, Switzerland) emulsion (Tanabe, Tokyo, Japan) was applied for $6 \mathrm{~h}$ before irradiation with narrowband continuous red light (wavelength $633 \mathrm{~nm}$, output light intensity $126 \mathrm{~mW} / \mathrm{cm}^{2}$ ) from light-emitting diodes (LEDs; Omnilux, Altrincham, Cheshire, UK) The individual LED cone angle and the spacing of the LEDs ensure uniformity of the beam for full coverage of the treatment area. The dimensions of the LED head active area were $150 \times 350 \mathrm{~mm}$ (length $\times$ width). Each lesion, plus a 5-mm margin of disease-free skin, was successively irradiated

\footnotetext{
${ }^{1}$ Department of Dermatology, Xijing Hospital, Fourth Military Medical University, Xi'an, China.

${ }^{2}$ Department of Dermatology, Armed Police Forces Beijing General Hospital, Beijing, China.

${ }^{3}$ Department of Dermatology, Hokkaido University Graduate School of Medicine, Sapporo, Japan.
} 
Table 1. Clinical Characteristics and Results of Patients with Cutaneous Pseudoepithliomatous Hyperplasia Treated with Topical Photodynamic therapy

\begin{tabular}{|c|c|c|c|c|c|c|c|}
\hline Patient & Age (years)/gender & Location & Lesion size $(\mathrm{mm})$ & $M A L(\%)$ & CID $\left(\mathrm{J} / \mathrm{cm}^{2}\right)$ & Result & Pain score (VAS) \\
\hline \multirow[t]{6}{*}{1} & \multirow[t]{6}{*}{$28 / \mathrm{F}$} & Right ankle & $3.5 \times 4.0$ & 5 & 113 & NR & 0 \\
\hline & & Right leg & $3.5 \times 2.5$ & 5 & 113 & NR & 0 \\
\hline & & Right leg & $2.0 \times 2.0$ & 10 & 113 & MR & 4 \\
\hline & & Right leg & $4.5 \times 5.0$ & 10 & 113 & NR & 1 \\
\hline & & Right leg & $2.0 \times 2.0$ & 15 & 113 & NR & 0 \\
\hline & & Right leg & $3.0 \times 2.5$ & 15 & 113 & NR & 1 \\
\hline \multirow[t]{6}{*}{2} & \multirow[t]{6}{*}{$37 / F$} & Left planta & $2.5 \times 3.0$ & 10 & 226 & NR & 0 \\
\hline & & Left planta & $3.5 \times 4.0$ & 10 & 226 & NR & 0 \\
\hline & & Left planta & $2.0 \times 1.0$ & 15 & 226 & NR & 0 \\
\hline & & Left foot, back & $4.0 \times 5.0$ & 15 & 226 & NR & 1 \\
\hline & & Left foot, back & $6.5 \times 6.0$ & 20 & 226 & NR & 1 \\
\hline & & Left ankle & $4.5 \times 5.5$ & 20 & 339 & NR & 1 \\
\hline \multirow[t]{4}{*}{3} & \multirow[t]{4}{*}{$45 / \mathrm{F}$} & Right leg & $1.5 \times 1.0$ & 25 & 226 & $\mathrm{MR}$ & 3 \\
\hline & & Right leg & $2.0 \times 2.0$ & 25 & 226 & MR & 3 \\
\hline & & Right leg & $3.0 \times 6.0$ & 30 & 226 & MR & 3 \\
\hline & & Right ankle & $4.0 \times 5.5$ & 30 & 339 & NR & 1 \\
\hline
\end{tabular}

MAL, methyl aminolaevulinate; CID, cumulative irradiation dose; MR, minimal response ( $<30 \%$ clearance); NR, no response; VAS, visual analogue scale.

at $10-15 \mathrm{~cm}$ distance for one to three sessions (total delivery light dose $=$ output light intensity $\times$ time: $113-339 \mathrm{~J} / \mathrm{cm}^{2}$ ), $15 \mathrm{~min}$ per session, 1 week apart. The procedures were handled according the manufacturers' recommendations. The light dose and MAL concentration were randomly used for the lesions. The lesions were photographed on a separate day of each week, so that neither the application of the cream nor the crusts affected assessment. Lesions were evaluated and rated by an observer blinded to the study protocol 3 months after the treatments. The lesions were scored as follows: complete response (CR, complete disappearance of lesions), minimal response ( $\mathrm{MR},<30 \%$ clearance), and no response (NR, no changes in size). Patients were scored on pain by a visual analogue scale (VAS; 0 , no pain; 10 , worst pain). ${ }^{8}$

The clinical characteristics and treatment response of each patient are summarized in Table 1. One PEH (patient 1) occurred after a scratch wound; two PEH cases (patients 2 and 3) were caused by a burn wound. The clinical response of one
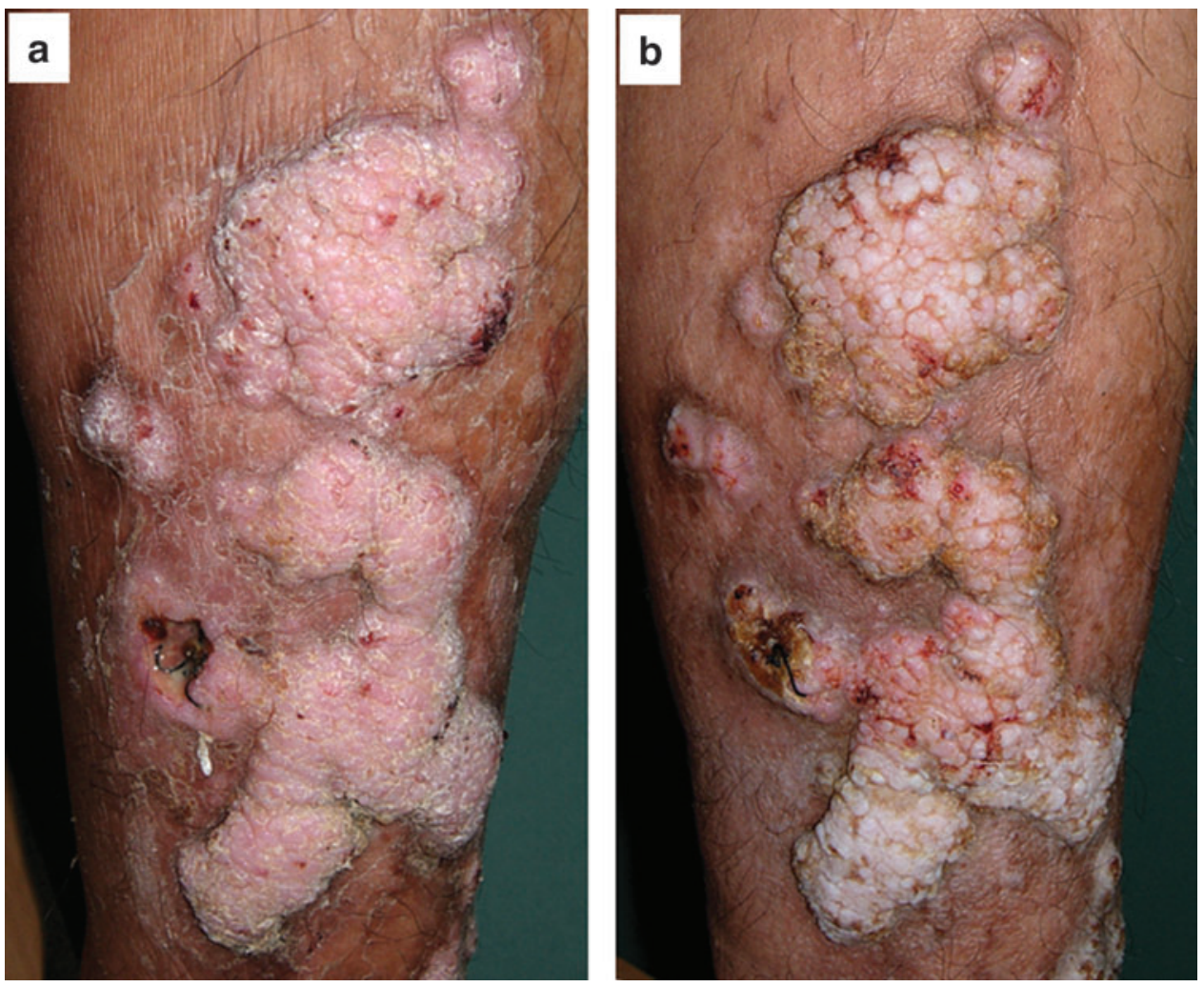

FIG. 1. Clinical response of cutaneous pseudoepithliomatous hyperplasia of leg treated with the ALA-PDT. Minimal response is shown after two treatments $\left(226 \mathrm{~J} / \mathrm{cm}^{2}\right) \quad$ (b), compared with the untreated lesions (a). 
representative case (patient 3 ) is shown in Fig. 1. Only 4 of 16 lesions clinically showed a minimal response. No response was seen in 12 of the 16 lesions, using either different cumulative doses or different concentrations of MAL. Although four lesions (patients 1 and 3) had a minimal response, changes were slight in diameter or thickness. This may be due to fibrosis in the dermis caused by PDT. The fibrosis was confirmed by histological examination.

\section{Discussion}

$\mathrm{PEH}$ is a type of exuberant proliferation of the epithelium with down-growth into the dermis occurring in a variety of settings. The pathogenesis still remains unclear. The histological features are similar with well-differentiated, invasive SCC, or may obscure an underlying malignant process, such as melanoma and granular cell tumour. There is no standard or optimal treatment: inappropriate invasive modalities such as cryotherapy and $\mathrm{CO}_{2}$ laser curettage leads to an extension of the lesion area. In the three cases studied, their occurrences were associated with skin wounds: underlying tumours were excluded by histological examination. They each successively received occlusive topical steroid therapy, cryotherapy, laser curettage, and topical 5FU. Unfortunately, these treatments were ineffective and even worsened the lesions. The patients refused surgical excision, since a large skin graft would be involved. Thus non-invasive PDT with its low-risk and positive cosmetic outcome was attempted. However, all the 16 lesions treated with topical PDT showed no complete response, even when given an increased photosensitizer or irradiation dose. Patients refused the second treatment.

This clinical trial indicates that topical MAL-PDT is not an effective therapy when applied to PEH after skin wounding. There are two possible factors associated with the poor response. One is the pathological features of PEH. It has been demonstrated that two mechanisms are involved in the clearance of a tumour and proliferating squamous cells. Epithelial neoplastic cells in AK, BD, and SCC may absorb much more photosensitizer than normal cells, leading to the elimination of the atypical cells. A study on the viral wart showed that PDT using an LED source achieved clearance rates of $81 \%$ (mean of 3.34 treatments). ${ }^{4}$ Complete clearance of periungula hand warts in 18 of 20 patients (30 of 40 warts) was achieved in a pilot study of PDT with ALA after a mean of 4.5 treatments. ${ }^{7}$ PDT leads to the release of inflammatory mediators, like interleukin 2 , interleukin $1-\beta$, and tumour necrosis factor$\alpha,{ }^{9}$ suggesting that another mechanism for the clearance of these cells is in the indirect stimulation of local immune responses in the lesion area. Although the PDT parameters that were employed for the viral wart or the epithelial neoplasms were also used for $\mathrm{PEH}$, all of the lesions responded poorly. This suggests that the lack of effectiveness could be attributed to the peculiar pathological pattern, not to the neoplastic or inflammatory epithelia proliferation of $\mathrm{PEH}$.

Another possibility for the lack of response is the thickness of the skin keratin layer in PEH. A previous study showed that two precancerous lesions, including oral verrucous hyperplasia $(\mathrm{OVH})$ and oral leukoplakia $(\mathrm{OL})$, responded well to topical $20 \%$ ALA-PDT; 8 of 8 OVH lesions achieved CR, 8 of 24 OL lesions showed CR. ${ }^{10}$ The major difference between $\mathrm{PEH}$ and OVH or OL is the thickness of oral mucosa and the cutaneous surface keratin layer. OL or OVH lesions with a thinner keratin layer achieved CR, which enabled more ALA to diffuse into the tumour tissue. ${ }^{10}$ This indicates that the poor response is associated with absorbance of the photosensitizing agent in $\mathrm{PEH}$, not with MAL concentration or illumination dose. It also indicates that therapeutic response seems not to be obviously related to treatment parameters. Nevertheless, there is a limitation with this study. As it was a small-sample case series, sampling error has to be involved in the therapeutic outcome and treatment parameters. Thus a large retrospective comparative follow-up study will be required for the re-confirmation of the relationship between the poor response and the parameters.

To some extent, pain reflects response during irradiation; patients ( 1 and 3 ) with greater pain at the same illumination are likely to have better response, as high pain scoring presents a severe destruction of local tumour tissue, regardless of lesion location. Although the patients with minimal response experienced mild pain compared with patients who had no response, the mean pain scores were still lower than the score of 6.4 of well-responded AK irradiated by LED. ${ }^{11}$

In conclusion, this case series suggests that topical PDT lacks effectiveness at the variable MAL concentrations and illumination dose. Thus we do not advocate the use of topical PDT for PEH patients with skin wounds. Besides removal of any underlying diseases, surgical excision is still the recommended first option in treating PEH.

\section{Author Disclosure Statement}

No competing financial interests exist.

\section{References}

1. Fu, X., Jiang, D., Chen, W., Sun, Bs. T., and Sheng, Z. (2007). Pseudoepitheliomatous hyperplasia formation after skin injury. Wound Repair Regen. 15, 39-46.

2. Campbell, S.M., and Curnow, A. (2008). Extensive vulval intraepithelial neoplasia treated with a new regime of systemic photodynamic therapy using meta-tetrahydroxychlorin (Foscan). J. Eur. Acad. Dermatol. Venereol. 22, 502-503.

3. Morton, C.A., McKenna, K.E., and Rhodes, L.E. (2008). Guidelines for topical photodynamic therapy: update. Br. J. Dermatol. 159, 1245-1266.

4. Smucler, R., and Jatsova, E. (2005). Comparative study of aminolevulic acid photodynamic therapy plus pulsed dye laser versus pulsed dye laser alone in treatment of viral warts. Photomed. Laser Surg. 23, 202-205.

5. Szeimies, R.M., Karrer, S., and Radakovic-Fijan, S. (2002). Photodynamic therapy using topical methyl 5-aminolevulinate compared with cryotherapy for actinic keratosis: a prospective, randomized study. J. Am. Acad. Dermatol. 47, 258-262.

6. Calzavara-Pinton, P.G., Venturini, M., and Sala, R. (2008). Methylaminolaevulinate-based photodynamic therapy of Bowen's disease and squamous cell carcinoma. Br. J. Dermatol. 159, 137-144.

7. Schroeter, C.A., Kaas, L., Waterval, J.J., Bos, P.M., and Neumann, H.A. (2007). Successful treatment of periungual warts using photodynamic therapy: a pilot study. J. Eur. Acad. Dermatol. Venereol. 21, 1170-1174.

8. Bird, S.B., and Dickson, E.W. (2001). Clinically significant changes in pain along the visual analog scale. Ann. Emerg. Med. 38, 639-643. 
9. Gollnick, S.O., Evans, S.S., and Baumann, H. (2003). Role of cytokines in photodynamic therapy-induced local and systemic inflammation. Br. J. Cancer 88, 1772-1779.

10. Lohmann, C.M., and Solomon, A.R. (2001). Clinicopathologic variants of cutaneous squamous cell carcinoma. Adv. Anat. Pathol. 8, 27-36.

11. Babilas, P., Knobler, R., and Hummel, S. (2007). Variable pulsed light is less painful than light-emitting diodes for topical photodynamic therapy of actinic keratosis: a prospective randomized controlled trial. Br. J. Dermatol. 157, $111-117$.
Address correspondence to: Qiang $\mathrm{Li}$ Department of Dermatology Xijing Hospital Fourth Military Medical University Chang Le Xi Lu 15 $X i^{\prime} a n$ Shaanxi 710032 China

E-mail: qiangli@fmmu.edu.cn; liqiangderma@gmail.com 